\title{
Risk of falls in elderly population in a Mexican community
}

Eduardo Mendez-Espinosa, Hector Riquelme-Heras*,Celina Gomez-Gomez, Raul Gutierrez-Herrera, Juan Barajas-Mora and Emmanuel Moreno-Bernal

Department of Family Practice, School of Medicine, UANL Monterrey, NL, Mexico

\begin{abstract}
Introduction: Falls are the most important source of morbidity and mortality for elders, becoming a serious public health problem because injuries that occur, in most cases respond to a mismatch between the person and his/her home environment.

Objective: To determine the environmental and personal factors that has risks for falls in older adults inan urban population of middle and low income.

Methodology: observational, cross-sectional study. The participants weresubjects $\geq 60$ years old, whose home is in the urban area of Monterrey, Nuevo Leon, Mexico, with medium and low income.

Results: A total of 132 people were surveyed, ranging from 60-93 years old; an average of 70.6, median 70; in the characteristics of housing, only $11.4 \%$ of the restrooms had available railings; of total households that had a ladder, only $32.6 \%$ had railings; $53 \%$ had a history of falling in the last year and $8.3 \%$ of them had a fracture.

Conclusions: Falls in this group of people with limited resources represent a major health risk because falls are a frequent event. There is no association between the use of psychotropic drugs and the presence of falls $(p<0.05)$. There is a significant association between the presence of disabilities with the presentation of falls $(p>0.05)$. We recommend that educational programs should be implemented to families with elderly relatives, oriented at adapting physical spaces andimprove the health of these people to avoid problems that cause disabilities that favor the presentation of falls.
\end{abstract}

\section{Introduction}

Falls in elderly people are a common public health problem that mainly affects the population of medium and low income. The community, wherethis population lives, is undergoing a demographic transition where elderly persons are growing in size;therefore, we consider that degenerative chronic problems will increase too and thus the risk of falls with the consequences of damage to their health. The prevalence of falls in the elderly varies from $30-50 \%$ with an annual incidence of $25-35 \%$. Between the $10-25 \%$ of falls in the elderly cause fractures, $5 \%$ require hospitalization [1]. Falls represent $30 \%$ the cause of death in people over 65 years [2]. Although falls carry a risk of injury to all persons, age, sex and health status can influence the type of injury and its severity. Age is one of the main risk factors for falls. The elderly is at highest risk of death ofserious injury from falls, and the risk increases with age. For example, in the United States $20-30 \%$ of older people who fall suffer moderate to severe injuries such as bruises, hip fractures or head trauma. The magnitude of the risk may be due at least in part, to physical, sensorial and cognitive impairments associated with aging [3], as well as the lack of adaptation of the environment to the needs of the elderly population. Another risk group is formed by children, who fall largely due to their state of development, their innate curiosity and their increasing level of independence, which leads them to take more risk behaviors. While inadequate adult supervision is a frequently cited factor, the circumstances are often complex and there are interactions with poverty and particularly hazardous environments [4]. Both genders are at risk of falling in all age groups and all regions. However, in some countries it has been observed that men are more likely to suffer fatal falls, while women suffer more nonfatal falls $[5,6]$. The elderly and young children are especially prone to falls and a greater severity of the resulting injury. Mortality rates and
Disability-Adjusted Life-Years (DALYs) are consistently higher in men worldwide. Among the possible explanations of this fact are the highest levels of risk behaviors and most dangerous work activities [2].

Extrinsic factorsare also common; they can be low light, loose rugs, stairs without railings, slippery floors, inclement weather (rain, rocks, snow, ice) or furniture placed improperly, leading to unsafe and dangerous environments to the elderly [7].

In a study in Uruguay [8], it found differentintrinsic risk factors such as: use of several drugs, comorbidities conditions and health problems as a result of the aging process itself. In old age, decreased muscle strength can upset the balance of the person, causing instability in the march. Furthermore, the presence of acute and chronic diseases, such as osteoporosis, for example, added to drug use can alter cognitive status, increasing the risk of falls, so it is important the assessment of these person.

This study aims to identify risk factors for falls that this group of population presents.

\section{General objective}

To know the environmental and personal factors that pose risks for

Correspondence to: Hector Riquelme-Heras, Department of Family Practice, School of Medicine, UANLMonterrey, NL, Mexico, Tel: 528180204829; E-mail: riquelme@doctor.com

Key words: falls, risks, adult, senior

Received: October 10, 2016; Accepted: November 17, 2016; Published: November 21, 2016 
falls in older adults in sub urban population of middle and low income.

\section{Methodology}

This is an observational, cross-sectional study, where study subjects were people $\geq 60$, whose home is in the urban area of Monterrey, Nuevo Leon, Mexico, with characteristics of medium and low income.

Prior informed consent of the participants was required. They answered an interview-questionnaire with sociodemographic information; presence of factors with potential risk of falls and personal history of falls.

The sample size obtained corresponds to 132 people of both genders; obtained from the formula of proportions with $95 \%$ confidence level with a value of $\mathrm{p}$ and q $50 \% 50 \%$ and an accuracy of $10 \%$.

Sampling was intentional to elderly people who attended the health care demand in primary careunits of the Ministry of Health and the Autonomous University of Nuevo Leon attended by family physicians.

Inclusion criteria were people $\geq 60$ years old, mentally competent and both genders.

The information obtained was integrated into a database and was processed by the SPSS18, (Statistical Package for the Social Sciences); measures of central tendency (mean) were calculated, and dispersion (standard deviation). Chi-square $\left(\chi^{2}\right)$ test for categorical variables ratios or relative frequencies were calculated.

\section{Results}

One hundred and thirty-twoolder adults were studied, whose age range was 60-93 years, mean 70.6, median 70, and mode was 60 . Standard deviation of 7.46 was calculated.Female gender predominated with $71.2 \%$; the rest corresponded to the male gender.

Marital status: stable union $43.2 \%$. Other participants were $35.6 \%$ widowhood, divorced and singlenessrepresented the $10.6 \%$.

In the type ofhealth insurancepredominated the free government Popular Insurance with 72\%; only 3\% did not have health insurance.

In relation to the characteristics of housing, only $11.4 \%$ of the bathrooms, had handrail; of total households, $58.3 \%$ of themwith ladder, $32.6 \%$ had handrails.In reference to education, the $24.2 \%$ of the respondents had no educational level; predominated complete and incomplete elementary school with $25.8 \%$, only $1.5 \%$ attended college studies. Household was the predominant occupation with $61.4 \%$. The $14.4 \%$ of elders lived alone; the rest with their partner and/ or children; $53.8 \%$ lived at home on one level. The 25.8 percent had some kind of disability, predominating movement limitation.Just over half of elderly, $54.4 \%$, kept lighting for their nocturnal movements.In relation to illnesses, $90.6 \%$ of those who participated in the study had a chronic disease, firstly mentioned diabetes mellitus in 43.8 percent of cases. When questioned about the antecedent of falls in the past year, $53 \%$ said this background; in $25 \%$ of cases, the fall occurred during the morning; $37 \%$ is related to accidents; $63 \%$ related to health condition. (ej. dizziness). Of all patients who suffered a fall, $8.3 \%$ had a fracture. The $62.9 \%$ of respondents said they had feelings of fear of falling; $81.1 \%$ reported not have received information about preventing falls, and who received it was granted mainly by his/her doctor.

It was found significant association between taking psychotropic medication and the presence of fall (Table 1). It was found significant association between the presence of some type of disability and the presentation of falls (Table 2). We found 2.13 OR for drugs use and 2.7
Table 1. Association between taking psychotropic medication and the presence of fall.

\begin{tabular}{|l|c|c|c|}
\hline Disability & \multicolumn{3}{|c|}{ Fallen in the last year } \\
\hline & Yes & No & Total \\
\hline Yes & 24 & 10 & 34 \\
\hline No & 46 & 52 & 98 \\
\hline Total & 70 & 62 & 132 \\
\hline $\mathrm{X}^{2}=5.66 ; \mathrm{p}=0.05$ & & & \\
\hline
\end{tabular}

Table 2. Association between the presence of some type of disability and the presentation of falls

\begin{tabular}{|l|c|c|c|}
\hline Disability & \multicolumn{3}{|c|}{ Fallen in the last year } \\
\hline & Yes & No & Total \\
\hline Yes & 9 & 4 & 13 \\
\hline No & 61 & 58 & 119 \\
\hline Total & 70 & 62 & 132 \\
\hline $\mathrm{X}^{2}=5.66 ; \mathrm{p}=0.05$ & & & \\
\hline
\end{tabular}

for disabilities. Fractures $6 \%$ and the present study $8 \%$

\section{Discussion}

According to the Clinical Practice Guidelines we found similarity in the frequency of falls in this age group with $53 \%$ found versus 30 $50 \%$ reported in the literature [1].

Comparing what was reported by WHO in 2016 [2], where it reports that about $20 \%$ of older adults have some serious injuries such as bruises, sprains or fractures, we found that $8.3 \%$ presented some type of fracture.

The poverty environment reported in different articles, as determinant risk factor for the presentation of falls is a characteristic of our studied population.

Similarly, the presence of incapacitating comorbidity and medication is compared with other studies that favor the falls of the elderly.

On the other hand, the characteristics of the house, such as lack of handrails, scalars without protection, lack of lighting, are results identified in other studies [3].

In relation to the greater presentation in the feminine gender is equal to the one reported by different authors [9].

Another study from Mexico identified risk factors for falls: architectural $24 \%$, furniture $16 \%$, equipment $22 \%$ and process $38 \%$. The areas with the highest number of risk factors identified were: common area $10 \%$, rooms $10 \%$ [10]. Our study reported: In relation to the characteristics of housing, only $11.4 \%$ of the bathrooms had handrail; of total households, $58.3 \%$ of them with ladder, $32.6 \%$ had handrails.

In a study conducted in the USA reported $23 \%$ of falls in the last year of the population studied. Compared to the present study it was $53 \%$ probably higher because the houses are not adapted for the elderly people in Mexico.

The relative risk (OR) in the USA study for falls with respect to drug use was 28.3 and for mental disorders of 5.0., and for disability $3.8 \%$. We found $2.13 \%$ OR for drugs use and $2.7 \%$ for disabilities. Fractures $6 \%$ and the present study $8 \%$ [11].

A recent review of literature (2013) reports a $30-40 \%$ frequency of falls in adults [12]. The range reported by the WHO is $30-40 \%$.

A primary care study reports a high incidence of both falls, mild 
(29\%) and severe falls (17\%). Severe falls were more common in nursing homes patients. More women than men experienced severe falls. There were positive associations between the number of falls and the total number of drugs [13].

\section{Conclusions}

Falls in older adults in this group of people with limited resources represent a significant health risk as fractures are a frequent event; 53 percent said they had suffered a fall in the last year, 8.3 percent suffered a fracture. Most people said they were afraid of falling because of the housing conditions and people characteristics. Disability is common in elderly, but what is relevant in this study is that most of the people surveyed had not received any kind of information for the prevention of falls or some other type of accidents.

We concluded that in this vulnerable group the sociodemographic factors, characteristics of housing, health conditions coupled with disabilities, lack of information for the prevention of falls are factors that affect the presentation of falls.

We recommend the implementation of educational programs for families with older adults, oriented to the adaptation of physical spaces and to improve the health of these people to avoid problems that cause disabilities that favor the presentation of falls. We need to consider that falls in the elderly can be prevented.

\section{References}

1. Prevention of Falls in the Elderly at the First Level of Care. Clinical Practice Guide. General Health Council Mexico.
2. World Health Organization, WHO (2016) Falls.

3. Silva Fhon JR, Coelho Fabrício Wehbe SC, Pereira Vendruscolo TR, Stackfleth R, Marques S, et al. (2012) Emollient and sweating with functional capacity. Revista Latino-Americana de Enfermagem 20: 927-934.

4. Sandoval L, Capuñay J, Varela L (1996) Falls in the Elderly: Study of a series of patients from an external medical practice at the Cayetano Heredia National Hospital. Revista Medica Herediana 7: 119-124.

5. Alfaro EV (2009) Prevention of falls in the elderly. Revista Médica de Costa Rica y Centroamérica 66: 353-355.

6. Oquendo DD, García ACB, Infante AP (1999) Incidence of falls in institutionalized older adults. Revista Cubana de Enfermería 15: 34-38.

7. Santamaría AL, Giménez PJ, Satorra TB, Orrio CN, Montoy MV (2015) Prevalence and factors associated with falls in older adults living in the community. Atención Primaria 47: 367-375.

8. Sgaravatti A (2011) Risk Factors and Assessment of Falls in the Elderly. Carta Geriátrico Gerontológica 4: 1-14.

9. Castillo DFE, Zapata HAR, Escobedo PS, Alonzo PA, Espino RA (2011) Incidence of falls in a sample of older adults of the University Unit of Rehabilitation of Mérida Yucatán. Revista Mexicana de Medicina Física y Rehabilitación 23: 8-12.

10. Rodríguez P, Ulises Pérez A; Sosa D, Domínguez G (2014) Extrinsic risk factors for falls in a home for older adults in Tabasco, Mexico. Archivos de medicina 10: 17.

11. Tinetti ME, Speechley M, Ginter SF (1988) Risk factors for falls among elderly persons living in the community. N Engl J Med 319: 1701-1707. [Crossref]

12. Ambrose AF, Paul G, Hausdorff JM (2013) Risk factors for falls among older adults: a review of the literature. Maturitas 75: 51-61. [Crossref]

13. Milos V, Bondesson Å, Magnusson M, Jakobsson U, Westerlund T, et al. (2014) Fall risk-increasing drugs and falls: a cross-sectional study among elderly patients in primary care. BMC Geriatr 14: 40. [Crossref]

Copyright: (C2016 Mendez-Espinosa E. This is an open-access article distributed under the terms of the Creative Commons Attribution License, which permits unrestricted use, distribution, and reproduction in any medium, provided the original author and source are credited. 